\title{
Endogenous Risk Measures
}

\author{
Moawia Alghalith \\ The University of the West Indies, St. Augustine, Trinidad \\ E-mail:malghalith@gmail.com \\ Received January 11, 2011; revised February 10, 2011; accepted February 28, 2011
}

\begin{abstract}
We present a methodology that allows endogenous derivation of the moments of the probability distributions. In doing so, we, present an alternative objective function and alternative concept of risk aversion. In addition, we show that the risk measure depends on the preferences. Moreover, we show that a higher level of risk aversion yields higher values of the risk measure.
\end{abstract}

Keywords: Risk, Risk Measures, Uncertainty

\section{Introduction}

In the absence of risk neutrality, probability measures have been subjectively determined by the individual. The risk-averse or risk-loving individual is assumed to subjectively determine the moments and the distribution of the risky variable. In addition, these distributions are assumed exogenous. That is, they are determined outside the stochastic model. Consequently, these results are ad hoc empirical and theoretical results. Examples include numerous theoretical and empirical results in the expected utility theory, arbitrage pricing theory, and stochastic finance.

For example, according to the expected utility theory, the probability distributions are still determined exogenously and independently of the individual's preferences (attitude towards risk), since preferences are determined by the form of the utility function [1]. That is, preferences determine the quantity of the decision variable but not the probability measure [2], among others). This appears to be counter-intuitive, since preferences should play a formal role in deriving the probability distributions. That is, it should be endogenously determined.

Other exogenous risk measures are extensively used in finance. The most prominent of these measures is the value at risk (VaR). The limitations of VaR are welldocumented and hence are needless to discuss (for example, see [3] and [4], among many others). Coherent risk measures and deviations measures are developed as an alternative to VaR. However, these measures are still exogenous and subjective measures. Even as exogenous measures, they have limitations (see [5] and [4] among others).
In this note, we develop a methodology that enables us to endogenously derive the moments of the probability distributions as the decision variables of the model. In so doing, we formally link the functional form of the objective function (attitude towards risk) to the derivation of the moments of the probability distributions. We use a model of decision-making under uncertainty as an example. Though the method is applicable to many other models, moreover, we present a more general and flexible model of decision-making under uncertainty, compared to the expected utility models (see [6]).

\section{The Model}

The conventional theory of the firm under uncertainty assumes that the firm maximizes the expected utility of the profit. However, the expected-utility-maximization objective does not describe the behavior of all firms. There is empirical evidence that suggests that the agent's behavior is inconsistent with the expected utility theory (see, for example, [7]). In the real world, the risk averse firm's objective is a mixture of profit-maxi- mization and risk-minimization. This is particularly true in the intermediate/long run. Consequently, we assume that the firm maximizes the function

$f(\pi)=\alpha E u(\pi)-\beta \operatorname{Var}(u(\pi))$ with respect to the moments of the distribution

$$
\max _{\sigma, \bar{p}} \int_{0}^{Y} f(\pi) \mathrm{d} y
$$

where $u$ is a bounded utility function $\left(u^{\prime}>0\right), \alpha \geq 0$ and $\beta \geq 0$ are parameters representing the firms motive/preferences. A higher value of $\beta$ indicates a higher 
level of risk-aversion and a more inclination to minimize risk, as opposed to profit maximizing. The opposite is true for $\alpha$. If the investor is extremely risk averse, $\alpha=0$ or $\beta=\infty$. Clearly, the EU theory is a special case of this theory when $\beta=0$. It is also a generalization of the mean-variance framework since our model does not require quadratic preferences. It is worth noticing, according to this model, $u^{\prime \prime}<0$ does not necessarily imply risk aversion; the converse is also true (risk aversion does not necessarily imply $\left(u^{\prime \prime}<0\right)$.

The profit function is specified by $\pi=p y-c(y)$, where $y$ is output, $c$ is the cost, $p$ is the random output price so that $p=\bar{p}+\sigma \varepsilon>0$, where $\sigma$ is the second moment, $\bar{p} \equiv E p$ and $\varepsilon$ is random (see [6]), among others). The assumption $p>0$ guarantees that $\bar{p}>0$ and $\sigma>0$. The output range is given by $y \in[0, Y]$ where $Y$ is the full-capacity output. For each fixed value of output $\hat{y}$.

The solution yields

$$
\begin{gathered}
\gamma E u^{\prime}(\pi) \varepsilon-2 \beta E u(\pi) u^{\prime}(\pi) \varepsilon=0, \\
\gamma E u^{\prime}(\pi)-2 \beta E u(\pi) u^{\prime}(\pi)=0,
\end{gathered}
$$

where $\gamma=\alpha+2 \beta E u(\pi)$ Thus, since we have two equations, optimal solutions can be obtained such that $\bar{p}^{*}$, $\sigma^{*} \in \arg \max (f(\pi))$. That is, the moments of the probability distribution can be obtained for each possible value of $y$. However, since we are we interested in measuring risk, we can modify the objective function to have the risk as the only decision variables

$$
\max _{\sigma} \int_{0}^{Y} f(\pi) \mathrm{d} y
$$

and the solution is

$$
\gamma E u^{\prime}(\pi) \varepsilon-2 \beta E u(\pi) u^{\prime}(\pi) \varepsilon=0 .
$$

\section{A Numerical Example}

Under the assumptions of normality, exponential utility $u(\pi)=-\mathrm{e}^{-\alpha \pi}$ (the usual assumption in the literature; see for example, [4]) and $\alpha=\gamma=\beta=1$ (other assumptions can also be used such as different utility functions or values of $\gamma, \alpha$ and $\beta$ ). Under the assumption of normality

$E f^{\prime}(\pi) \varepsilon=E f^{\prime \prime}(\pi) \operatorname{cov}(\pi, p)=y^{2} \sigma^{2} E f^{\prime \prime}(\pi)$. Given $y=10, \bar{p}=1, c(y)=9$, we obtain $\sigma=0.146$.

We used the assumptions of normality and exponential utility to simplify the calculations. Other functional forms and distributions are feasible using computer programming.

\section{References}

[1] M. Yaari, "The Dual Theory of Choice under Risk," Econometrica, Vol. 55, No. 1, 1987, pp. 95-115. doi: $10.2307 / 1911158$

[2] A. Sandmo, "On the Theory of the Competitive Firm Under Price Uncertainty," American Economic Review, Vol. 61, No. 1, 1971, pp. 65-73.

[3] R. E. Bailey, "The Economics of Financial Markets," Cambridge University Press, Cambridge, 2005.

[4] R. Elliott and E. Kopp, "Mathematics of Financial Markets," Springer, New York, 2005.

[5] R. Elliott and T. Siu, "On Risk Minimizing Portfolios under a Markovian Regime-Switching Black-Scholes Economy," Annals of Operations Research, forthcoming 2010. doi:10.1007/s10479-008-0448-5

[6] M. Alghalith, "New Economics of Risk and Uncertainty: Theory and Applications," Nova Science Publishers Inc., New York, 2007.

[7] B. J. Cohen, "Is Expected Utility Theory Normative for Medical Decision Making?" Medical Decision Making, Vol. 16, 1996, pp. 1-6. doi: $10.1177 / 0272989 \times 9601600101$ 